\title{
Étude ethnobotanique d'espèces ligneuses des savanes sèches au Nord-Togo : diversité, usages, importance et vulnérabilité
}

\author{
Bériname Badjaré $^{(1)}$, Kouami Kokou ${ }^{(2)}$, Nadédjo Bigou-laré ${ }^{(3)}$, Dabitora Koumantiga ${ }^{(4)}$, \\ Ayitre Akpakouma ${ }^{(5)}$, Macomba Bétidé Adjayi ${ }^{(5)}$, Georges Abbévi Abbey ${ }^{(1)}$ \\ (1) Université de Lomé. École Supérieure d'Agronomie. Département d'Économie et Sociologie rurales. 05 BP 643. Lomé 05 \\ (Togo).E-mail : beriname.badjare@gmail.com \\ (2) Université de Lomé. Faculté des Sciences. Laboratoire de Botanique/Écologie. BP 1515. Lomé (Togo). \\ (3) Université de Lomé. Faculté des Sciences économiques et de Gestion. Laboratoire de Recherche sur le Management des \\ Organisations. BP 1515. Lomé (Togo) - Institut Supérieur Privé de Management (Togo). \\ (4) Université de Lomé. Faculté des Sciences. BP 1515. Lomé (Togo). \\ (5) Université Laval. 2425, rue de l'agriculture. G1V 0A6 Québec, Québec (Canada).
}

Reçu le 28 mai 2017, accepté le 15 mai 2018, mis en ligne le 15 juin 2018.

Cet article est distribué suivant les termes et les conditions de la licence CC-BY (http://creativecommons.org/licenses/by/4.0/ deed.fr)

Description du sujet. Au Nord-Togo, particulièrement en milieu rural, les populations utilisent des biens d'espèces ligneuses et des services écosystémiques pour satisfaire des besoins multiples. Dans un environnement marqué par une déforestation accrue, se développe une pression anthropique croissante avec une incidence sur la vulnérabilité et la valorisation de ces ressources.

Objectifs. Cette étude sur la diversité et les usages des ligneux permet d'apprécier la richesse floristique des terroirs et d'évaluer, à partir de quatre paramètres, la vulnérabilité des espèces répertoriées et connues des populations en zone de savane sèche.

Méthode. L'approche méthodologique est basée sur des enquêtes ethnobotaniques et socio-économiques menées auprès de 14 groupes ethniques par interview individuel et au sein de «focus groups». Les analyses ont porté sur des calculs de fréquences et d'indices de valeurs d'usage et d'importance des espèces répertoriées.

Résultats. Au total, 149 espèces ligneuses utiles ont été identifiées et réparties en 45 familles. Neuf principaux usages reconnus sont relevés : alimentaire (69 espèces), médicinal ( 91 espèces), bois-énergie (57 espèces), fourrager (57 espèces), artisanal (37 espèces), soins hygiéniques (36 espèces), bois de service (40 espèces), rituel ou magico-spirituel (35 espèces) et cosmétique (26 espèces). Ces usages portent essentiellement sur les feuilles, racines, fruits, graines, fleurs, bois et écorces. Sur l'ensemble des espèces répertoriées, 42 (28\%) présentent un risque élevé de vulnérabilité, celle-ci étant évaluée sur base de quatre paramètres : la fréquence de citation, le nombre d'usages différents, l'organe collecté et le mode de collecte.

Conclusions. L'étude montre que l'utilisation des espèces ligneuses varie selon le milieu géographique et la valeur d'importance des espèces explique la vulnérabilité de celles-ci dans la savane sèche.

Mots-clés. Plantes ligneuses, services écosystémiques, utilisation, durabilité, savanes, Togo.

Ethnobotanical study of woody species of the dry savannah in northern Togo: diversity, uses, importance and vulnerability

Description of the subject. In northern Togo, particularly in rural areas, the populations use ecosystem services to meet multiple needs within a context of deforestation and increasing human pressure that threatens natural resources.

Objectives. Based on people's knowledge, this study studied the diversity and the vulnerability of multipurpose tree species in the dry savannah zone of Togo, using four parameters of vulnerability.

Method. The methodology was based on semi-directive ethnobotanical and socioeconomic surveys within 14 ethnic groups, through individual interviews and focus group discussions. Analyses included both an index of uses and importance values of species identified and frequency calculations. 
Results. 149 tree species, corresponding to 45 families, were cited as being useful for the investigated peoples; nine categories of purpose were identified: food ( 69 species), medicinal ( 91 species), wood as an energy source (57 species), livestock feeding (57 species), artisanal (37 species), hygiene (36 species), service wood (40 species), ritual (35 species), and cosmetic (26 species). Various parts of a tree can be used: leaves, roots, fruits, seeds, flowers, wood and bark. A total of 42 tree species (28\%) presented a high level of vulnerability, which was assessed on the basis of four parameters: the frequency of citation, the number of different uses, the organs collected and the method of harvesting.

Conclusions. The study revealed that the use of forest trees species depends on their geographical area and their importance value explains the vulnerability of these species in the Savannah region.

Keywords. Woody plants, ecosystem services, uses, sustainability, savannas, Togo.

\section{INTRODUCTION}

En Afrique, il est largement admis que les ressources naturelles présentent, pour les populations locales, un intérêt socio-économique certain et leur permettent de satisfaire leurs besoins fondamentaux. Pour plusieurs groupes ethniques sur le continent, elles contribuent, entre autres, à la réduction de la pauvreté et à la sécurité alimentaire (Loubelo, 2012). Ces ressources sont des biens publics classés dans la catégorie des biens communs (Yelkouni, 2012). De la description et l'analyse de la végétation, Kent \& Cooker (2003) et Luck et al. (2009) ont qualifié la biodiversité, y compris les services écosystémiques, de biens publics très étendus nécessitant la protection. Ils estiment que l'état des forêts est le reflet des actifs capitalisés, du fait qu'il permet, a priori, une appréciation des valeurs futures du patrimoine ou du potentiel en ressources forestières considérées à la fois comme des actifs forestiers et des placements de diversification susceptibles de générer des plus-values ou des richesses pour les communautés. La perte de ces ressources peut d'ailleurs provoquer un affaiblissement du potentiel local et de la capacité des communautés tributaires de la forêt à en tirer des revenus et de la nourriture. La présence des ligneux dans les espaces cultivés et les jachères constitue une caractéristique fondamentale des paysages agraires au sud du Sahara (Wala, 2001). Dans cet espace, les communautés locales récoltent en général et directement de nombreux produits forestiers (Kokou et al., 2006; Sop et al., 2011). Malgré le rôle crucial joué par les espèces ligneuses dans le développement socio-économique et dans les réponses aux besoins multiples des populations et sur les questions de durabilité, le taux élevé de déforestation sur le continent africain demeure inquiétant (FAO, 2012).

$\mathrm{Au}$ Togo, la couverture forestière s'établit à 24,4\% de l'étendue nationale (MERF, 2016), soit environ 1362840 ha constitués d'îlots et de massifs forestiers qui font l'objet d'une règlementation sur le territoire national, ainsi que des écosystèmes forestiers des terroirs villageois dont le mode de gestion est généralement communautaire. Dans un contexte global de changement climatique impactant en particulier les disponibilités alimentaires, la résilience des systèmes sociaux et environnementaux est mise à l'épreuve. Plusieurs études ont été menées dans le pays sur les plantes utiles, mais elles sont focalisées soit sur un type précis d'usage, soit sur une espèce spécifique, soit encore sur la valeur socioculturelle des plantes alimentaires dans une dynamique de préservation (Kokou et al., 2009 ; Akpavi et al., 2013 ; Atato et al., 2013 ; Atakpama et al., 2014). Face à la pression anthropique sur les espèces ligneuses utiles et leur importance dans la vie socio-économique des membres des communautés locales, qui généralement font de petits déplacements et quelquefois de grands parcours visiblement sans contrainte majeure afin de prélever des organes de plantes, il est nécessaire d'explorer la diversité de ces espèces ainsi que les fonctions et usages qu'elles revêtent pour ces communautés. Les enjeux actuels liés à l'utilité ou l'attribution d'une capacité aux services écosystémiques à répondre aux besoins des communautés soulèvent des questions :

- le contexte socioculturel explique-il en partie la composition floristique des socio-écosystèmes dans la savane sèche au Nord-Togo ?

- la valeur d'importance de l'espèce d'arbre forestier explique-t-elle sa vulnérabilité ?

Le manque d'informations scientifiques et socioéconomiques, en particulier sur la distribution spatiale des espèces ligneuses utiles, les usages socioculturels des biens et services écosystémiques, les valeurs d'usage ainsi que les valeurs d'importance des espèces sont sans doute parmi les contraintes de valorisation des ressources forestières d'une part (Zohoun et al., 2002 ; Dourma, 2008 ; Kébézikato, 2014) et, d'autre part, de contribution à une gestion durable du milieu naturel (Barbier, 2007 ; Brahic \& Terreaux, 2009a ; Brahic \& Terreaux, 2009b). Cet article analyse la composition floristique des socio-écosystèmes ainsi que les fonctions et les usages d'espèces ligneuses utiles connues par les communautés dans la savane sèche. Il aborde également l'importance socioéconomique et la vulnérabilité des espèces dans cet espace géographique du Nord-Togo. 


\section{MATÉRIEL ET MÉTHODES}

\subsection{Zone d'étude}

Cette étude est réalisée au Nord-Togo dans les zones écologiques I et II (Figure 1) correspondant à une végétation de savane sèche (Agboh \& Badjaré, 2007). Cette zone se compose de trois régions administratives que sont la région des Savanes, la région de la Kara et celle de la Centrale. Ces trois régions couvrent une superficie totale de $33658 \mathrm{~km}^{2}\left(8533 \mathrm{~km}^{2}\right.$ pour la Savane, $11625 \mathrm{~km}^{2}$ pour la Kara et $13500 \mathrm{~km}^{2}$ pour la Centrale), soit environ 59,3\% du territoire national. La savane sèche jouit d'un climat de type soudanien comprenant une saison sèche et une saison pluvieuse. La pluviométrie annuelle est de $1201,69 \mathrm{~mm}$ et la température moyenne annuelle de $27,7^{\circ} \mathrm{C}$ (Badjaré, 2012). Les températures extrêmes varient entre $39^{\circ} \mathrm{C}$ et $17^{\circ} \mathrm{C}$ en saison sèche et entre $34^{\circ} \mathrm{C}$ et $22^{\circ} \mathrm{C}$ en saison des pluies (Agboh \& Badjaré, 2007). La population, qui est en grande majorité rurale, s'établissait à 1598164 habitants en 2010 (INSEED, 2011), avec un

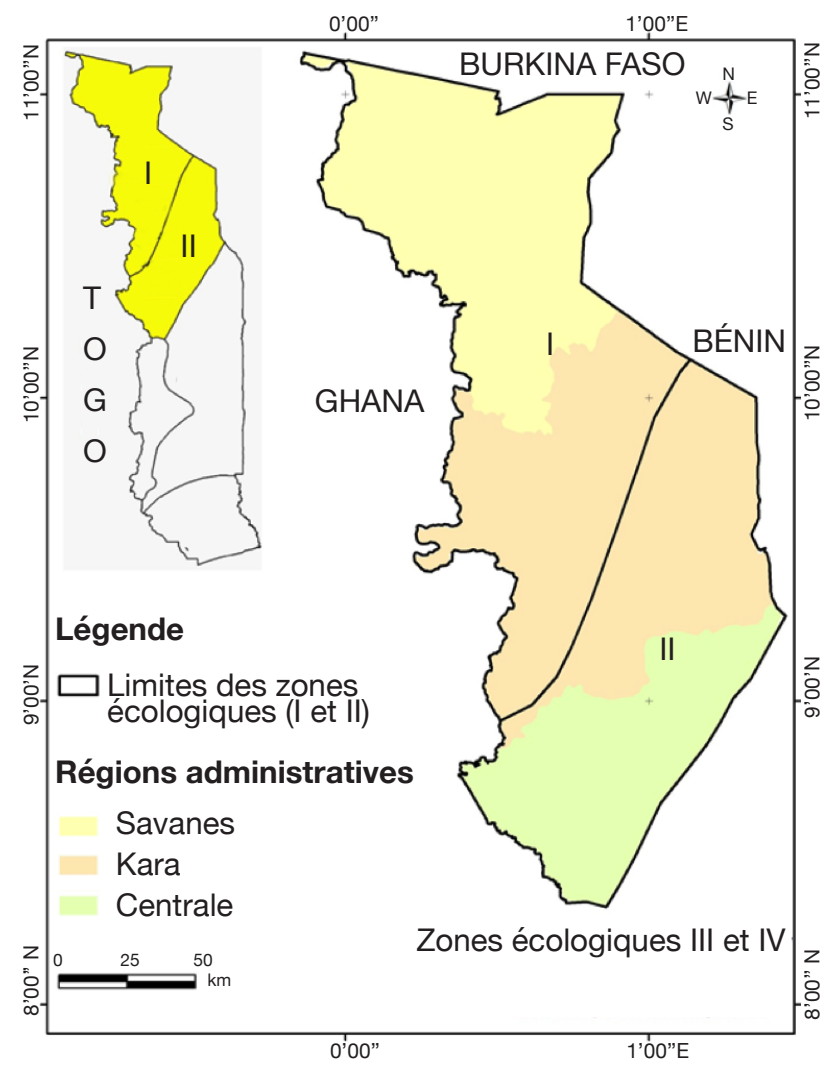

Figure 1. Carte de la zone de l'étude - Map of the study area.

Sources : DGSCN, 2010 ; données de terrain, 2015 ; carte Topo-Togo IGN, France. Auteurs : Badjaré B. \& Doumantiga D., 2017 - DGSCN, 2010 ; field data, 2015 ; map Topo-Togo IGN, France. Authors : Badjaré B. \& Doumantiga D., 2017. taux d'accroissement annuel moyen estiméà 2,3\% entre 2011 et 2031, selon les perspectives démographiques $\mathrm{du}$ Togo (INSEED, 2015). Les villes de cette zone connaissent ces dernières années une forte urbanisation et une pression démographique en lien avec la pression intense sur les ressources agricoles et la végétation.

\subsection{Méthodes de collecte des données}

Échantillonnage et source des données. L'échantillonnage stratifié (Atakpama et al., 2012 ; Zabouh, 2014) a été adopté. Ainsi, deux strates ont été distinguées ; une première est constituée des trois régions cibles du Togo que sont les Savanes, la Kara et la Centrale, dont les deux premières sont constituées de 12 préfectures couvertes par cette étude et auxquelles s'ajoute la zone reliant la préfecture d'Assoli localisée dans la région de la Kara et celle de Tchaoudjo dans la région Centrale. La deuxième strate se compose de 36 cantons choisis de manière à couvrir l'ensemble des groupes ethniques présents dans chacune des préfectures. Des villages sont ensuite choisis au hasard pour la conduite des enquêtes. De manière globale, il a été adopté une méthodologie d'enquêtes ethnobotaniques semidirectives par interview individuel et au sein des focus groups. Au total, 558 personnes ont été enquêtées dont 173 personnes dans la région des Savanes réparties dans les préfectures de Cinkansé, Tône, Kpendjal, Tandjoare et Oti, et 354 personnes dans la région de la Kara réparties dans les préfectures de la Kéran, Doufelgou, Kozah, Binah, Assoli, Bassar et Dankpen. Dans la partie est des préfectures d'Assoli et de Tchaoudjo, 31 personnes ont été enquêtées. La répartition par sexe donne des proportions de $42-58 \%$, respectivement pour les femmes et les hommes ; ceci se justifie par le fait que généralement, dans les zones rurales, les femmes sont plus ou moins réservées et rarement candidates à des exercices de réponse aux questions. L'enquête a pris en compte toute personne âgée d'au moins 20 ans, vivant dans les zones ciblées, sans distinction de sexe, ni d'âge. Les personnes intérrogées appartiennent à 14 groupes ethniques qui ont permis d'enregistrer 4021 citations d'espèces ligneuses dans la zone de l'étude. L'enquête porte essentiellement sur les espèces ligneuses de savane sèche.

Enquêtes et indicateurs d'analyse. La démarche d'enquête dans son ensemble a permis de rassembler les informations quantitatives et qualitatives sur :

- la composition floristique des ligneux utiles identifiés dans les zones cibles;

- les fonctions, les usages possibles et spécifiques des différentes espèces utiles ainsi que des services fournis aux populations par ces ressources forestières ;

- l'importance socio-économique des espèces ligneuses répertoriées ; 
- les paramètres d'appréciation de la vulnérabilité des espèces connues et sollicitées par les populations dans la zone de l'étude.

Au cours des enquêtes pour la collecte de données, certaines espèces citées et décrites en langue locale par les communautés n'ont pas pu être identifiées immédiatement. Pour ce faire, des sorties de terrain avec des enquêtés ont permis d'identifier ces espèces et de complèter leur identité taxonomique.

\subsection{Traitements et analyse des données ethnobotaniques}

Les échantillons des espèces répertoriées dans la savane sèche et collectées sont identifiés avec des noms scientifiques attribués à l'aide de documents de référence, notamment :

- la flore analytique du Togo (Brunel et al., 1984) ;

- la flore du Bénin (Akoegninou et al., 2006) ;

- la flore arbustive et forestière des zones sèches d'Afrique de l'Ouest (Arbonnier, 2002).

Les espèces non identifiées sur le terrain ont été prélevées et apportées au laboratoire aux fins d'identification taxonomique. Le système de classification phylogénétique des angiospermes (APG II, 2003) redéfinissant les familles a été utilisé pour ce niveau taxonomique.

À partir du traitement des données ethnobotaniques et des données socio-économiques et démographiques, il a été réalisé des analyses sur:

- les valeurs d'usage et les valeurs d'importance des espèces ;

- la disponibilité des espèces ou l'abondance de cellesci ;

- l'évaluation de la vulnérabilité des espèces de communautés végétales des zones ciblées dans la savane sèche au Nord-Togo.

Les différents paramètres pour le calcul des valeurs d'importance et d'usage sont les suivants.

La fréquence de citation $(F)$ d'une espèce correspond au rapport entre le nombre d'enquêtés $(n)$ ayant cité l'espèce et le nombre total d'enquêtés $(N)$ :

$$
F=\frac{n}{N} \times 100 \text {. }
$$

La valeur d'importance (VIsp) de l'espèce représente le rapport entre le nombre d'usages différents pour l'espèce $(v i)$ et le nombre d'usages différents pour l'ensemble des espèces répertoriées $\left(\sum v i\right)$ :

$$
V I s p=\frac{v i}{\sum v i} \times 100 .
$$

Deux indices d'usage sont également calculés, notamment le nombre d'usage de l'espèce (NUsp) et la valeur d'usage de l'espèce (VUsp).

Le nombre d'usage de l'espèce correspond à la somme des nombres de citation d'usage par organe de l'espèce. NUorgane est le nombre de citations pour un organe spécifique de la plante par l'ensemble des enquêtés :

$$
N U s p=\sum N U \text { organes } .
$$

La valeur d'usage de l'espèce correspond au rapport entre $N U s p$ et la somme totale des nombres d'usage de toutes les espèces $(\Sigma N U s p i)$. La formule utilisée est la suivante :

$$
V U s p=\frac{N U s p}{\sum N U s p i} .
$$

L'espèce ayant la valeur la plus élevée est celle dont l'usage est le plus reconnu.

Le calcul des valeurs d'usage basé sur le nombre des usages et le nombre de personnes ayant cité une espèce donnée permet de connaitre les espèces les plus importantes pour une communauté (Albuquerque et al., 2006). Ainsi, plus la valeur d'usage est élevée, plus l'espèce est importante.

Pour l'analyse de similarité de présence des espèces sur les espaces géographiques étudiés, ainsi que des usages connus par les différentes communautés organisées autour des 14 groupes ethniques enquêtés, un ajustement a été réalisé par la méthode Whittaker's Index of Association (1972) basée sur l'analyse de gradient. Cette méthode a permis d'apprécier l'étendue des liens et d'établir des relations entre les variables - notamment et, d'une part, les zones géographiques pour ce qui est de la similarité de présence des espèces sur les terroirs et, d'autre part, les groupes ethniques ou groupes ethnoculturels répartis sur ces différentes zones pour ce qui est de l'appréciation de la similarité des usages au sein des différentes communautés.

\subsection{Analyse de la vulnérabilité liée aux usages et à la demande}

Pour faire ressortir les principales espèces potentiellement vulnérables par la forte demande (ramassage, cueillette ou coupe d'organes) en biens et services écosystémiques dans les localités ciblées, le calcul de l'indice du risque de vulnérabilité des espèces (IV) a été adapté de Betti (2001) et Traoré et al. (2011). Ainsi, le risque de vulnérabilité, dont l'évaluation repose essentiellement sur les sollicitations des espèces et de leurs organes et pas directement sur la rareté ou l'abondance de l'espèce, a été calculé sur la base de quatre paramètres, qui sont : 
- la fréquence de citation de l'espèce ;

- le nombre de catégorie d'usages dans lequel se retrouve l'espèce ;

- le mode de prélèvement ;

- l'organe utilisé.

Ces paramètres représentent des indicateurs majeurs des pressions et menaces exercées sur les espèces ligneuses sollicitées dans la zone. Une échelle de 1 à 3 a été attribuée à ces paramètres (Tableau 1). Une valeur de 1 désigne un faible risque de vulnérabilité de l'espèce pour les paramètres indiqués, une valeur de 2 représente un risque moyen de vulnérabilité et une valeur de 3 caractérise une espèce à risque élevé de vulnérabilité. Lorsque plusieurs parties d'une plante sont sollicitées dans un usage, seule la partie qui a la plus grande valeur issue de l'échelle de vulnérabilité est prise en compte dans le calcul des indices (Traoré et al., 2011 ; Birregah, 2016).

Sur la base des informations contenues dans le tableau 1, l'indice de vulnérabilité est calculé à travers la formule :

$$
I V=\frac{P 1+P 2+P 3+P 4}{4}
$$

En définitive, la vulnérabilité forte est attribuée pour des indices totaux strictement supérieurs à 6 , celle caractérisée de moyenne est attribuée pour des indices totaux compris entre 3 et 6 et la vulnérabilité relativement faible correspond aux indices compris entre 1 et 3 .

\section{RÉSULTATS}

\subsection{Composition floristique en espèces ligneuses utiles dans la savane sèche au Nord-Togo}

Composition floristique et répartition. Les investigations ethnobotaniques menées ont permis de dénombrer 149 espèces ligneuses utiles (Annexe 1). Les espèces inventoriées dans le cadre de cette étude se répartissent en 45 familles. Le spectre biologique (Figure 2) fait ressortir une

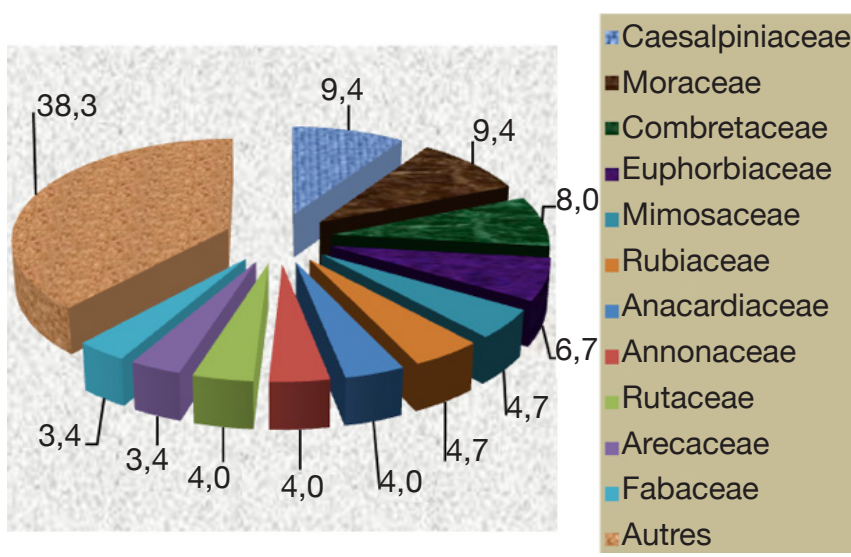

Figure 2. Répartition des espèces au sein des familles Distribution of species within families.

dominance des Caesalpiniaceae $(9,4 \%)$, Moraceae $(9,4 \%)$, Combretaceae $(8,0 \%)$, Euphorbiaceae $(6,7 \%)$, Mimosaceae $(4,7 \%)$, Rubiaceae $(4,7 \%)$, Anacardiaceae (4,0\%), Annonaceae $(4,0 \%)$ et Rutaceae $(4,0 \%)$. Les autres familles représentent $38,3 \%$ des taxons.

Composition floristique suivant les zones couvertes. $\mathrm{Au}$ niveau des différentes zones couvertes, la distribution des fréquences spécifiques et l'analyse de similarité montrent clairement que l'utilisation dominante des espèces ligneuses varie selon le milieu géographique. Dans un intervalle de similarité de 40 à $78 \%$ représentant le pourcentage d'espèces ligneuses citées et connues dans une zone donnée par rapport au nombre total répertorié dans la savane sèche, des similarités de présence sur les espaces géographiques et d'usages des espèces sont établies pour les différentes préfectures ciblées en lien avec les groupes ethniques en présence.

Le groupe I se subdivise en deux sous-groupes GI A et GI B. Le groupe II comprend également deux sous-groupes (GII A et GII B). Tous les sous-groupes présentent quelques similarités (Figure 3).

En somme, la distribution des espèces ligneuses utiles au sein des groupes ethniques représentant des groupes ethnoculturels montre que le regroupement

Tableau 1. Paramètres majeurs pris en compte pour le calcul de l'indice de vulnérabilité (adapté de Betti, 2001 ; Traoré et al., 2011) - Major parameters considered for vulnerability index calculation (adapted from Betti, 2001; Traoré et al., 2011).

\begin{tabular}{llll}
\hline Paramètre retenu & Faible (échelle = 1) & Moyenne (échelle = 2) & Forte (échelle = 3) \\
\hline Fréquence de citation : P1 & $\mathrm{P} 1<5 \%$ & $5 \% \leq \mathrm{P} 2<15 \%$ & $\mathrm{P} 3 \geq 15 \%$ \\
Nombre d'usages : P2 & $\mathrm{P} 2<2$ & $2 \leq \mathrm{P} 2 \leq 4$ & $\mathrm{P} 2 \geq 5$ \\
Organe végétal utilisé : P3 & Feuille, latex & Fruit & Bois, graine, écorce, racine, fleur \\
Mode de collecte de l'organe : P4 & Ramassage & - & Cueillette, coupe \\
\hline
\end{tabular}




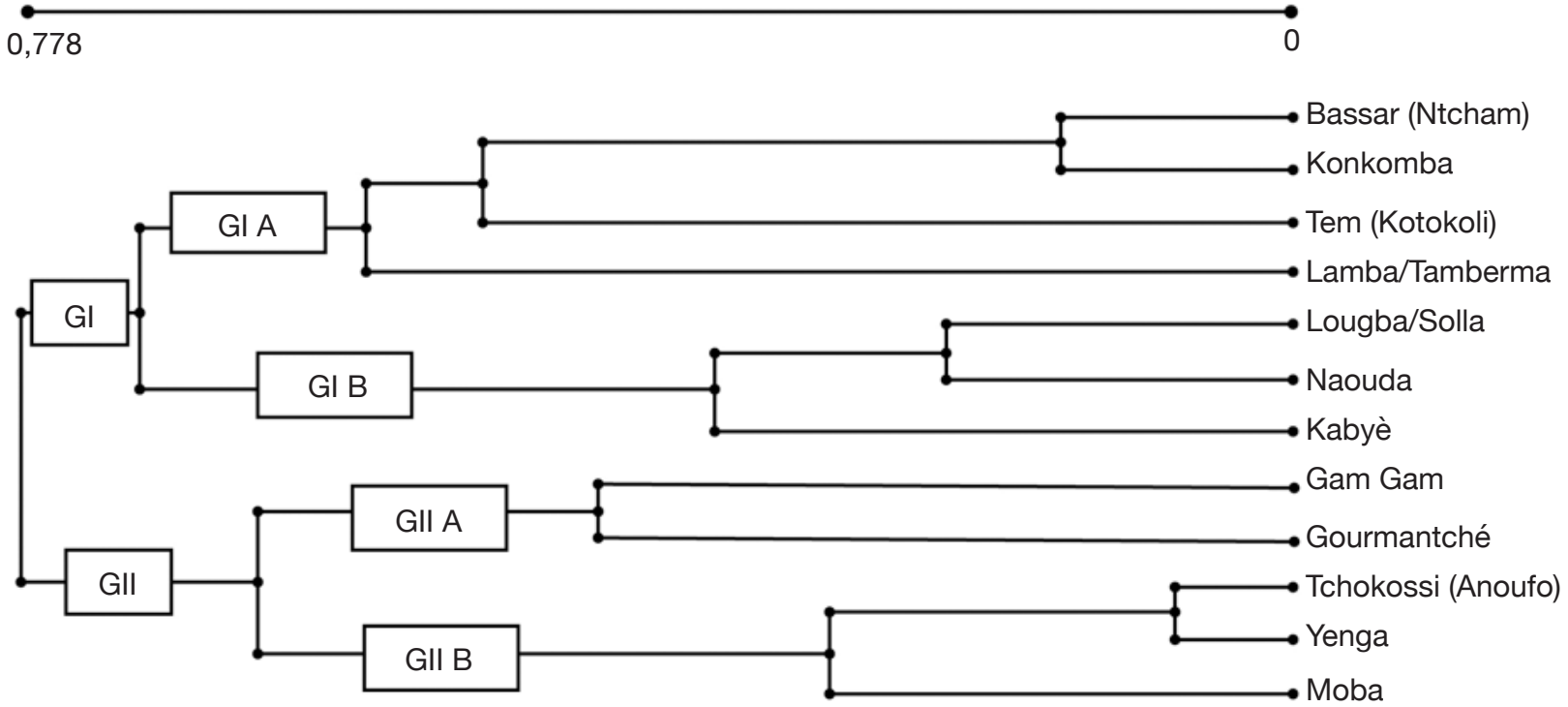

Figure 3. Dendrogramme ajusté par Whittaker's Index of Association montrant la relation entre groupes ethniques et espèces ligneuses connues par les enquêtés et répertoriées - Dendrogram adjusted by Whittaker's Index of Association showing the relationship between ethnic groups and woody species known to respondents and indexed.

Ntcham ou Bassar, Konkomba, Tem ou Kotokoli et Lamba/Tamberma (GI A) présente la plus forte diversité d'espèces ligneuses, supérieure à $40 \%$ (61 espèces sur 149 répertoriées). Il est suivi du sous-groupe GII A au sein des groupes ethniques Gam Gam et Gourmanché, du sous-groupe GI B regroupant les ethnies Lougba/ Solla, Naouda et Kabyè et de GII B avec les groupes Tchokossi ou Anoufo, Yenga et Moba au sein desquels sont respectivement identifiées 57,52 et 48 espèces ligneuses.

Distribution rang-fréquences des espèces. La courbe de distribution des fréquences spécifiques présente une allure décroissante (Figure 4). Parkia biglobosa (24,0\%), Mangifera indica (23,5\%), Adansonia digitata $(23,0 \%)$ et Vitellaria paradoxa $(22,2 \%)$ présentent une fréquence relative de plus de $20 \%$, d'où la dominance de l'utilisation de ces espèces dans la savane sèche au Nord-Togo. Six espèces ligneuses ont une fréquence relative supérieure à $13 \%$. Il s'agit de Blighia sapida (15\%), Elaeis guineensis $(14,3 \%)$, Borassus aethiopum (13,6\%), Ceiba pentandra $(13,6 \%)$, Diospyros mespiliformis $(13,4 \%)$ et Vitex doniana $(13,3 \%)$.

Il apparait, par ordre décroissant, 14 espèces ayant une fréquence relative comprise entre 7 et $12 \%$ dont, par exemple, Azadirachta indica, Prosopis africana, Moringa oleifera, Pterocarpus erinaceus, Khaya senegalensis, Ficus sur, Afzelia africana et Anacardium occidentale. Au total, 125 espèces ligneuses utiles répértoriées ont des fréquences relatives inférieures ou égales à $6 \%$.

\subsection{Usages des espèces ligneuses dans la savane sèche au Nord-Togo}

Utilisation des principales espèces ligneuses répertoriées. La typologie des utilisations possibles fait ressortir des 4021 citations enregistrées neuf principaux usages reconnus par les populations de la savane sèche. L'ensemble des citations donnent, comme fréquence, $34,0 \%$ pour l'utilisation alimentaire (avec 69 espèces inventoriées), 15,4\% pour les besoins pharmaceutiques ou médicinaux (avec 91 espèces identifiées), 12,2\% pour les besoins en bois-énergie (avec 57 espèces ligneuses), 10,0\% pour l'utilisation fourragère (57 espèces répertoriées), $7,4 \%$ pour la destination artisanale (avec 37 espèces identifiées), $6,8 \%$ pour l'hygiène corporelle (36 espèces répertoriées), $6,7 \%$ pour le bois de service (40 espèces identifiées), $4,6 \%$ pour les besoins rituels ou magicospirituels (35 espèces identifiées) et 2,9\% pour les besoins cosmétiques (avec 26 espèces relevées).

Les principales espèces alimentaires citées sont: Mangifera indica, Vitellaria paradoxa, Parkia biglobosa, Adansonia digitata et Lannea microcarpa. Les espèces ligneuses fréquemment citées comme espèces sollicitées pour la pharmacopée ou la médecine traditionnelle sont, entre autres, Pseudocedrela kotschyi, Sterculia setigera, Gardenia erubescens, Strychnos innocua, Securidaca longepedunculata et Khaya senegalensis. Pour les besoins en boisénergie, les espèces principalement mentionnées dans cette zone sont Acacia albida, Acacia gourmaensis, Albizia lebbeck, Anacardium occidentale, Annona 
Badjaré B., Kokou K., Bigou-laré N. et al.

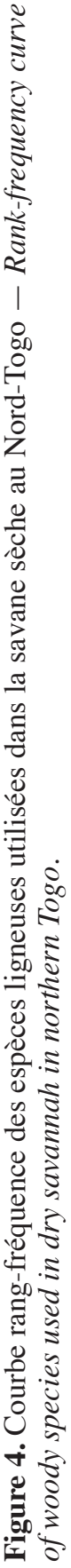

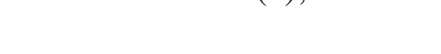


ondulata et Azadirachta indica. Les espèces sources de fourrage sont fréquemment Eucalyptus camaldulensis, Pterocarpus erinaceus, Stereospermum kunthianum, Vitellaria paradoxa, Daniellia oliveri et Borassus aethiopum. Pour l'hygiène corporelle, sont principalement utilisées: Prosopis africana, Azadirachta indica, Diospyros mespiliformis, Blighia sapida, Eucalyptus tereticornis et Acacia gourmaensis. Des usages rapportés aux valeurs magico-spirituelles et aux rituels (rites spirituels, notamment funéraires) concernent, entre autres, les espèces Adansonia digitata, Parkia biglobosa, Afzelia africana, Ficus thonningii, Milicia excelsa, Prosopis africana et Sterculia setigera. Dans le domaine cosmétique, les espèces Vitellaria paradoxa, Azadirachta indica et Lannea acida sont utilisées avec le beurre et les huiles produits à partir d'organes des deux premières plantes. Les feuilles de Lannea acida sont utilisées pour la coloration des mains ou des pieds, ainsi que pour le maquillage. D'autres espèces citées à des fins cosmétiques ou esthétiques sont Afraegle paniculata, Alchornea hirtella, Anacardium occidentale, Blighia sapida, Bombax costatum et Ficus abutilifolia.

Valeurs d'usage des principales espèces sollicitées. Suivant les valeurs d'usage (Tableau 2), il apparait que sur les 20 premières essences utiles répertoriées, Vitellaria paradoxa $(7,77)$ est l'espèce dont l'usage est le plus connu dans la savane sèche et donc l'arbre le plus sollicité. D'autres espèces, entre autres, Adansonia digitata, Parkia biglobosa, Mangifera indica, Borassus aethiopum Vitex doniana, Diospyros mespiliformis, Moringa oleifera et Anacardium occidentale figurent parmi ces essences utiles identifiées dans la zone.

\subsection{Valeurs d'importance des espèces ligneuses dans la savane sèche}

Les valeurs d'importance des espèces (VIsp ; Annexe 2) font ressortir que Vitellaria paradoxa, Parkia biglobosa, Borassus aethiopum, Pterocarpus erinaceus, Elaeis guineensis et Azadirachta indica ont une valeur d'importance de 1,00, soit neuf usages sur un total de neuf répertoriés. Les espèces Blighia sapida, Anogeissus leiocarpus et Ceiba pentandra ont une valeur d'importance de 0,88 , soit huit usages sur neuf. Les essences forestières Bombax costatum, Diospyros mespiliformis, Hyphaene thebaica, Khaya senegalensis, Lannea acida, Mangifera indica et Prosopis africana ont une valeur d'importance de 0,77, soit sept usages sur neuf. Les espèces ligneuses Afzelia africana, Eucalyptus camaldulensis, Tamarindus indica et Terminalia glaucescens ont six usages sur neuf, soit une valeur d'importance de 0,67. Adansonia digitata, Antiaris africana, Daniellia oliveri, Gmelina arborea, Lannea barteri et Vitex doniana ont une
Tableau 2. Valeurs (VUsp) et nombres (NUsp) d'usages des principales espèces utiles identifiées par cette étude - Values (VUsp) and numbers (NUsp) of uses of the main useful species identified by this study.

\begin{tabular}{lll}
\hline Espèce & NUsp & VUsp \\
\hline Vitellaria paradoxa & 382 & 7,77 \\
\hline Adansonia digitata & 311 & 6,33 \\
\hline Parkia biglobosa & 306 & 6,23 \\
\hline Mangifera indica & 271 & 5,51 \\
\hline Azadirachta indica & 213 & 4,33 \\
\hline Elaeis guineensis & 203 & 4,13 \\
\hline Borassus aethiopum & 154 & 3,13 \\
\hline Pterocarpus erinaceus & 153 & 3,11 \\
\hline Vitex doniana & 153 & 3,11 \\
\hline Ceiba pentandra & 144 & 2,93 \\
\hline Prosopis africana & 135 & 2,74 \\
\hline Blighia sapida & 131 & 2,66 \\
\hline Diospyros mespiliformis & 122 & 2,48 \\
\hline Khaya senegalensis & 118 & 2,40 \\
\hline Moringa oleifera & 109 & 2,21 \\
\hline Ficus sur & 73 & 1,48 \\
\hline Senna siamea & 70 & 1,42 \\
\hline Afzelia africana & 68 & 1,38 \\
\hline Anacardium occidentale & 63 & 1,28 \\
\hline Citrus sinensis & 63 & 1,28 \\
\hline
\end{tabular}

valeur d'importance de 0,56 . Les principales espèces présentant quatre usages sur neuf sont Acacia albida, Acacia gourmaensis, Anacardium occidentale, Annona senegalensis et Bridelia ferruginea. Celles ayant trois usages sur neuf sont principalement Afraegle paniculata, Albizia lebbeck, Balanites aegyptiaca, Citrus sinensis, Delonix regia et Detarium microcarpum.

\subsection{Vulnérabilité des espèces ligneuses dans la savane sèche}

Le calcul des indices du risque de vulnérabilité (IV) basé sur les sollicitations des espèces montre que 42 des 149 espèces répertoriées, soit $28,19 \%$, présentent un risque élevé de vulnérabilité (indice de vulnérabilité strictement supérieur à 6) contre 55,70 \% d'espèces à risque de vulnérabilité moyen (indice compris entre 3 et 6 ) et $16,11 \%$ des espèces ont un risque de vulnérabilité relativement faible (indice compris entre 1 et 3 ). Les 42 espèces ligneuses à vulnérabilité forte sont présentées dans le tableau 3. 
Tableau 3.Espèces ligneuses à vulnérabilité forte - Woody species with high vulnerability.

\begin{tabular}{|c|c|}
\hline Espèce & Indice de vulnérabilité (IV) \\
\hline Adansonia digitata & 9,00 \\
\hline Mangifera indica & 9,00 \\
\hline Parkia biglobosa & 9,00 \\
\hline Vitellaria paradoxa & 9,00 \\
\hline Afzelia africana & 7,75 \\
\hline Anogeissus leiocarpus & 7,75 \\
\hline Azadirachta indica & 7,75 \\
\hline Blighia sapida & 7,75 \\
\hline Bombax costatum & 7,75 \\
\hline Borassus aethiopum & 7,75 \\
\hline Ceiba pentandra & 7,75 \\
\hline Daniellia oliveri & 7,75 \\
\hline Diospyros mespiliformis & 7,75 \\
\hline Elaeis guineensis & 7,75 \\
\hline Gmelina arborea & 7,50 \\
\hline Hyphaene thebaica & 7,50 \\
\hline Khaya senegalensis & 7,50 \\
\hline Prosopis africana & 7,50 \\
\hline Pterocarpus erinaceus & 7,50 \\
\hline Tamarindus indica & 7,50 \\
\hline Tectona grandis & 7,50 \\
\hline Vitex doniana & 7,50 \\
\hline Antiaris africana & 7,00 \\
\hline Eucalyptus tereticornis & 7,00 \\
\hline Lannea acida & 7,00 \\
\hline Lannea barteri & 7,00 \\
\hline Terminalia glaucescens & 7,00 \\
\hline Anacardium occidentale & 6,50 \\
\hline Annona senegalensis & 6,50 \\
\hline Carica papaya & 6,50 \\
\hline Citrus sinensis & 6,50 \\
\hline Cocos nucifera & 6,50 \\
\hline Detarium microcarpum & 6,50 \\
\hline Ficus polita & 6,50 \\
\hline Ficus sur & 6,50 \\
\hline Gardenia erubescens & 6,50 \\
\hline Moringa oleifera & 6,50 \\
\hline Pseudocedrela kotschyi & 6,50 \\
\hline Psidium guajava & 6,50 \\
\hline Pteleopsis suberosa & 6,50 \\
\hline Sarcocephalus latifolius & 6,50 \\
\hline Senna siamea & 6,50 \\
\hline
\end{tabular}

\section{DISCUSSION}

\subsection{Composition floristique et influence sur les connaissances ethnobotaniques locales}

Dans les milieux naturels, chaque type de végétation est caractérisé par une structure et une diversité floristique marquée par des dominances de familles, de genres ou d'espèces (Jaffré \& Veillon, 1991). Certains groupes ethniques ont des similarités dans les usages spécifiques connus de biens et services écosystémiques. Par exemple, les Moba, les Gourmantche, les Yenga et les Gam Gam localisés dans l'extrême nord du pays et les Konkonba au centre-ouest considèrent Sterculia setigera comme une espèce ayant un pouvoir magico-spirituel, alors que chez les Naouda, c'est plutôt Afzelia africana qui est doté d'un tel pouvoir (Koumantiga et al., 2013). De plus, dans la tradition Moba et Gourmantche, les espèces Adansonia digitata et Parkia biglobosa sont utilisées dans les cérémonies spirituelles et funéraires. Très clairement, les réalités socioculturelles et la promiscuité entre les groupes ethniques de l'espace géographique ciblé auraient véritablement une incidence sur les connaissances des espèces ligneuses (Rakotoarimanana et al., 2008 ; Atakpama, 2010) et les choix opérés par les différents groupes ethniques sur la base, entre autres, du « savoir ancien ». Ce savoir est généralement légué par les ascendants et porte surtout sur les connaissances des vertus des plantes servant, par exemple, d'intrants ou de recettes en médecine traditionnelle (Zabouh, 2014), les valeurs magico-spirituelles de certaines espèces et les totems qu'il convient de respecter au sein des communautés. D'autres opportunités comme les liens de mariage renforcent les relations interethniques qui restent dynamiques dans le temps et dans l'espace avec des influences certaines sur les habitudes, les comportements et les connaissances endogènes (Van Auken, 2009). Les connaissances des usages des espèces et la diversité dans la composition floristique, qui permettent de rendre compte du degré de connexion entre groupes ethniques, est particulièrement sous l'influence de conditions édaphiques, anthropiques et de variabilité hydrologique ou climatique (Mahamane et al.,2007 ;Adjonou et al.,2009; Naito \& Cairns, 2011 ; D'Odorico \& Bhattachan, 2012). Dans ces conditions, les informations relatives à l'état d'exploitation ou d'utilisation des ressources forestières, notamment les espèces ligneuses, les organes sollicités ou les services écosystémiques fournis, demeurent des sources précieuses pour une gestion concertée et durable (Rives, 2012). Les résultats d'analyse de la diversité des espèces ligneuses utiles dans la savane sèche togolaise corroborent ceux énoncés par la FAO (2011) dont il ressortait que les espèces ligneuses utiles les plus fréquentes dans les zones écologiques I et II sont 
Vitellaria paradoxa, Parkia biglobosa, Adansonia digitata, Borassus aethiopum, Anogeissus leiocarpa, Chochlospermum tinctorim, Detarium microcarpum, Guiera senegalensis, Pterocarpus erinaceus, Diospyros mespiliformis, integrifolia, Daniellia oliveri, Khaya senegalensis et Vitex doniana.

La distribution des espèces ligneuses utiles citées par les différents groupes ethnoculturels enquêtés fait ressortir que les Tem, les Konkonba, les Moba, les Kabyè, les Bassar, les Gourmantché, les Naouda, les Lamba et les Tchokossi ont connaissance d'un nombre d'espèces représentant plus de $35 \%$ du nombre total répertorié (149 espèces). De plus, l'analyse de la distribution des fréquences spécifiques montre que l'utilisation dominante des espèces ligneuses varie selon le milieu géographique et donc selon le groupe ethnique en présence, en rapport avec ses connaissances générales et/ou spécifiques de l'espèce ou de ses organes sollicités.

\subsection{Importance et utilisation des biens et services écosystémiques d'espèces ligneuses}

Pour nombre d'auteurs, la conservation de la biodiversité constitue un support pour la fourniture de biens et services écosystémiques, y compris les bénéfices économiques qu'ils génèrent (DalyHassen et al., 2010). Généralement, des organes de nombreuses espèces ligneuses sont utilisés en réponse à des besoins multiples des communautés humaines, surtout en milieu rural où ces espèces font partie des moyens d'existence des populations avec des valeurs sociales et économiques attachées à chacune d'elle (Daly-Hassen et al., 2005 ; Neffati et al., 2015). Il s'agit essentiellement de services d'approvisionnement ou de prélèvement qui assurent le maintien de la société rurale (Rives, 2012), y compris la restauration des terres dégradées (Chazdon, 2008).

Dans la savane sèche au nord du Togo, neuf principaux usages de biens et services écosystémiques d'espèces forestières sontreconnus par les populations. Ces usages portent essentiellement sur les feuilles, racines, fruits, graines, fleurs, bois et écorces. De la littérature, il ressort une distinction entre les usages qui altèrent les écosystèmes, par exemple en modifiant leur diversité, et ceux qui génèrent des avantages sans leur porter atteinte (Salles, 2010). Dans les usages des biens et services écosystémiques, il est relevé une dynamique des relations de compétition pour les ressources (Brunelle et al., 2013), avec une évolution des usages dans le temps et dans l'espace qui ne sont pas sans conséquence à moyen ou long terme sur la durabilité des socio-écosystèmes. Des auteurs reconnaissent que la valeur d'importance d'une plante peut expliquer sa préservation et l'attribution d'une place spéciale dans le système cultural ou de gestion du terroir (Guillemette, 2014).

L'utilisation de Adansonia digitata et Parkia biglobosa est commune à 13 zones sur les 14 couvertes au Nord-Togo dans la savane sèche. L'usage de Mangifera indica et de Vitellaria paradoxa est commun respectivement à 12 et à 10 zones. Les espèces Diospyros mespiliformis et Lannea microcarpa sont utilisées communément dans 8 zones, tandis que Blighia sapida, Borassus aethiopum et Ceiba pentandra se révèlent liées à sept zones. Sont communes à cinq ou six zones dans leur utilisation, les espèces Azadirachta indica, Bombax costatum, Elaeis guineensis et Vitex doniana. Dans une logique de durabilité, des acteurs locaux détenteurs de pouvoirs et de potentialités peuvent fondamentalement contribuer à l'utilisation rationnelle des biens et services écosystémiques d'espèces ligneuses. Dans la savane sèche togolaise, l'organisation communautaire n'est pas encore suffisamment structurée pour contribuer à une gestion efficiente et durable des ressources forestières. Les structures locales en place, notamment des comités de développement à la base, ont des capacités limitées pour s'approprier les outils et mettre en œuvre les méthodes éprouvées de gestion durable des ressources forestières. Cette situation est exacerbée par les différences socioculturelles qui ont une incidence sur les processus de valorisation des savoirs et savoir-faire locaux, rendant les communautés, dans une certaine mesure, encore plus vulnérables. Dans ces conditions, il est important de proposer aux communautés cibles des réponses qui, d'une part, tiennent compte du contexte socioculturel, y compris les besoins d'usages spécifiques des espèces et qui, d'autre part, intègrent des modules d'apprentissage sur les mécanismes adéquats de gestion des espèces ligneuses (Daly-Hassen et al., 2005 ; Rives, 2012).

$\mathrm{Au}$ Nord-Togo, les actions correctrices doivent être menées en considérant désormais les territoires villageois comme l'échelle de référence pour la promotion de la bonne gouvernance dans la gestion des ressources forestières. Il s'agira de :

- capitaliser les connaissances adaptées, entre autres, sur les pratiques d'agriculture dite intelligente contribuant au pouvoir économique des communautés cibles ;

- renforcer les capacités organisationnelles, institutionnelles et techniques des principales couches sociales et des structures locales en vue d'une appropriation des systèmes simples d'aménagement et d'affectation des terres, y compris l'agroforesterie ;

- développer des programmes contribuant à la restauration et à la gestion des écosystèmes naturels pour l'optimisation de leurs services. 


\subsection{Vulnérabilité d'espèces ligneuses utiles dans la savane sèche}

Les informations sur les sollicitations des espèces et la nature des organes prélevés renseignent sur la vulnérabilité de ces ressources (Traoré et al., 2011). Sur la base des paramètres retenus, les valeurs de l'indice de vulnérabilité identifient 28,19\% des taxons comme présentant un risque élevé de vulnérabilité. Au regard de la grande diversité des usages des espèces ligneuses et leur importance socio-économique, sources de défis liés à la vulnérabilité et à la dégradation des espaces boisés, la démarche «Sustainable Livelihood Approach » ou SLA est recommandée dans une première phase pour permettre un diagnostic global devant orienter toute intervention corrective, en particulier dans la gestion forestière et le développement rural intégré dans la savane sèche. Le cadre théorique de l'approche SLA défend en effet l'idée d'une influence positive ou négative des processus et des structures ou des institutions, à différents niveaux, sur le contexte de vulnérabilité ainsi que sur les actifs et la vulnérabilité des individus. Cette approche est une démarche d'amélioration de la compréhension des moyens de subsistance des populations pauvres, comme la majorité de celles qui peuplent le Nord-Togo et qui font largement recours, entre autres, aux organes d'espèces forestières ligneuses.

Pour la zone vulnérable du Nord-Togo, il est recommandé, dans une seconde phase d'intervention, de promouvoir des pratiques sylvicoles innovantes basées en particulier sur des opérations à petite échelle (Miller et al., 1995). Différentes méthodes sylvicoles inspirées de Blaser (1996) sont proposées pour stimuler, d'ici 2030, au Nord-Togo, la restauration forestière. Ces méthodes seront sans doute utiles pour le maintien des populations des 42 espèces supposées vulnérables par cette étude. De plus, pour la zone cible, la protection des espèces sur les terres privées devra être stimulée à travers des mesures de motivation visant les propriétaires et encourageant le volontarisme. Cette option est d'ailleurs partagée par Ozier (1994) et Irwin \& Wigley (1992) qui pensent que la protection des espèces sur les terres privées pourrait être assumée par les propriétaires privés, à la condition qu'ils puissent le faire à un cout raisonnable et dans un réseau de coopération. Il est également recommandé aux autorités la mise en place d'une stratégie de communication qui visera l'appropriation, l'implication, le changement de comportement et surtout la participation des populations à la mise en œuvre des activités de restauration des paysages forestiers.

Par ailleurs, il serait important d'élaborer pour les espèces ligneuses qui entrent dans les activités économiques (exploitation du bois), des normes d'exploitabilité (diamètre minimal d'exploitabilité, tarif de cubage) afin de garantir la reconstitution des populations exploitées.

\section{CONCLUSIONS}

Au Nord-Togo, il existe une multitude d'usages des espèces ligneuses et l'utilisation des biens et services écosystémiques issus de ces ressources forestières varie selon le milieu géographique. Pour la durabilité des écosystèmes et la promotion de la «forêt objectif », donc susceptible de rendre des services à la communauté, les aménagements forestiers devront suffisamment tenir compte des spécificités d'usages connus par les populations locales, des valeurs d'usage et d'importance des espèces ligneuses, des valeurs des biens et services écosystémiques ainsi que des situations de vulnérabilité des espèces, eu égard aux fréquentes et multiples sollicitations dont elles font l'objet dans un contexte marqué, selon l'opinion, par une rareté de substituts proches, surtout pour les plantes alimentaires. Quelques espèces à usage médicinal comme Azadirachta indica semblent, par endroit et au sein de certains groupes ethniques, disposer de substituts relatifs constitués de molécules synthétiques utilisées pour des soins de santé. Ainsi, pour l'intérêt des générations présentes et futures, la sensibilisation des populations quant aux risques de vulnérabilité des espèces utiles, l'émergence d'alternatives et de substituts de produits de plantes ligneuses à travers, entre autres, des pratiques innovantes d'agroforesterie avec des conséquences directes sur les politiques publiques et la promotion de nouvelles sources d'énergies constituent de véritables pistes de solutions. Les résultats de cette étude contribueront au développement de programmes intégrés de restauration ou de préservation des ressources d'espèces ligneuses au Togo et, par conséquent, sur l'économie nationale. Pour renforcer les mécanismes de réponses aux besoins de gestion durable de ces ressources, l'analyse de l'incidence des considérations socioculturelles sur la demande en biens et services d'espèces ligneuses utiles, y compris les relations de pouvoirs des parties prenantes, est indispensable. Elle renseignerait sur les facteurs naturels endogènes liés à ces ligneux et contribuerait à la sécurité humaine au sein des groupes ethniques. En définitive, pour la préservation des écosystèmes forestiers dans la savane sèche et la diversification des sources d'approvisionnement en biens et services écosystémiques, le niveau d'implication des communautés locales dans les programmes reste un défi et une priorité. 


\section{Bibliographie}

Adjonou K., Bellefontaine R. \& Kokou K., 2009. Les forêts claires du Parc national Oti-Kéran au Nord Togo : structure, dynamique et impacts des modifications climatiques récentes. Sécheresse, 20(1), 1-10.

Agboh N. \& Badjaré B., 2007. Critères de stratification du Togo en zones homogènes pour la recherche agronomique. Rapport d'activités. Lomé : Institut Togolais de Recherche Agronomique.

Akoegninou A., Van der Burg W.J. \& Van der Maesen L.J.G., 2006. Flore analytique du Bénin. Leiden, Pays-Bas: Backhuys Publisher.

Akpavi S. et al., 2013. Valeur socio-culturelle des plantes alimentaires : un facteur de préservation. Eur. Sci. J., 9(32), 383-395.

Albuquerque U.P., Lucena R.F.P., Monteiro J.M. \& Florentino A.T.N., 2006. Evaluating two quantitative ethnobotanical techniques. Ethnobot. Res. Appl., 4, 5160 .

APG II, 2003. An update of the Angiosperm Phylogeny Group classification for the orders and families of flowering plants: APG II. Bot. J. Linn. Soc., 141, 399436.

Arbonnier M., 2002. Arbres, arbustes et lianes des zones sèches d'Afrique de l'Ouest. $2^{\mathrm{e}}$ ed. Paris: Musée National d'Histoire Naturelle ; Versailles, France : Quæ.

Atakpama W., 2010. Étude des formations à Sterculia setigera Del. dans la zone écofloristique I du Togo: aspects structural et socio-économique. DEA : Université de Lomé (Togo).

Atakpama W. et al., 2012. Ethnobotanical knowledge of Sterculia setigera Del. in the Sudanian zone of Togo (West Africa). ISRN Bot., 2012, ID 723157.

Atakpama W. et al., 2014. Moringa oleifera Lamarck (Moringaceae) : une ressource phytogénétique à usage multiple. Rev. Cames, 2(1), 6-14.

Atato A. et al., 2013. Espèces lianescentes à fruits comestibles du Togo. Fruits, 67, 353-368.

Badjaré B., 2012. Risques climatiques et durabilité des productions de semences certifiées de maïs et de riz au Togo. Master de recherche : Université de Lomé (Togo).

Barbier S., 2007. Influence de la diversité, de la composition et de l'abondance des essences forestières sur la diversité floristique des forêts tempérées. Thèse de doctorat: Université d'Orléans (France).

Betti J.L., 2001. Vulnérabilité des plantes utilisées comme antipaludiques dans l'arrondissement de Mintom au sud de la réserve de biosphère du Dja (Cameroun). Syst. Geogr. Plants, 71(2), 661-678.

Birregah B.W., 2016. Usages endogènes des plantes dans la préfecture de Doufelgou : exploitations, menaces et méthodes de conservation. Mémoire: Université de Lomé (Togo).

Blaser J., 1996. Sylvicultural considerations of listing timber species in Appendices I, II and III of CITES. In: Second meeting of the Timber Working Group, Convention on International Trade in Endangered Species of Wild Fauna and Flora, October 1996, Panama.

Brahic E. \& Terreaux J.P., 2009a. Pourquoi et comment estimer la valeur économique de la biodiversité forestière ? Rendez-vous Techn. ONF, 25-26, 63-68.

Brahic E. \& Terreaux J.P., 2009b. Évaluation économique de la biodiversité. Méthodes et exemple pour les forêts tempérées. Versailles, France : Quæ.

Brunel J.F., Hiepko P. \& Scholz H., 1984. Flore analytique du Togo: phanérogames. Berlin, Allemagne: Botanischer Garten und Botanisches Museum BerlinDahlem.

Brunelle A., Minckley T.A., Delgadillo J. \& Blissett S., 2013. A long-term perspective on woody plant encroachment in the desert southwest, New Mexico, USA. J. Veg. Sci., 25, 829-838.

Chazdon R.L., 2008. Beyond deforestation: restoring forests and ecosystem services on degraded lands. Science, 320(5882), 1458-1460.

Daly-Hassen H., Campos P., Ovando P. \& Chebil A., 2005. Economic analysis of cork oak woodland natural regeneration in the region of Iteimia, Tunisia. In: Proceedings of the International Congress on Cork plantations, factories and traders. The past, present and future of the cork business, 16-18 February 2005, Palafrugell, Spain.

Daly-Hassen H., Gader G. ～\& Potthast M.C., 2010. Une approche économique pour choisir les options d'adaptation des forêts au changement climatique: application à la forêt de chêne liège en Tunisie. In : Actes du XIV Congrès forestier mondial, 7-11 septembre 2015, Durban, Afrique du Sud.

D’Odorico P. \& Bhattachan A., 2012. Hydrologic variability in dryland regions: impacts on ecosystem dynamics and food security. Phil. Trans. R. Soc. B, 367(1606), 31453157.

Dourma M., 2008. Les forêts claires à Isoberlinia sp. dans la zone soudanienne du Togo: écologie, régénération naturelle et impacts humains. Thèse de doctorat : Université de Lomé (Togo).

FAO, 2011. Plan d'action forestier national du TogoPhase 1 (PAFN 1-Togo) 2011-2019. Rome : FAO.

FAO, 2012. Rapport de l'organisation des Nations Unies pour l'alimentation et l'agriculture sur la situation des forêts en 2012. Rome : FAO.

Guillemette E., 2014. Caractérisation et suivi des attributs multiressources de la Forêt habitée du Massif dans un contexte d'aménagement durable. Essai. Maîtrise en science forestière: Université de Laval, Québec (Canada).

INSEED (Institut National de la Statistique et des Études Économiques et Démographiques), 2011. Togo Quatrième recensement général de la population et de l'habitat au Togo (2009-2011). Lomé : Direction Générale de la Statistique et de la Comptabilité Nationale. 
INSEED (Institut National de la Statistique et des Études Économiques et Démographiques), 2015. Perspectives démographiques du Togo 2011-2031. Rapport édition 2015. Lomé : INSEED.

Irwin L.L.\& Wigley T.B., 1992. Conservation of endangered species. J. For., 90(8), 27-30.

Jaffré T. \& Veillon J.M., 1991. Étude floristique et structurale de deux forêts denses humides sur roches ultrabasiques en Nouvelle-Calédonie. Paris: Museum National d'Histoire Naturelle.

Kébézikato A.B. et al., 2014. Distribution et structure des parcs à Adansonia digitata L. (baobab) au Togo (Afrique de l'Ouest). Afr. Sci., 10(2), 434-449.

Kent M. \& Cooker P., 2003. Vegetation description and analysis. A pratical approach. Hoboken, NJ, USA: John Wiley and Son.

Kokou K. et al., 2006. Diagnostic socioéconomique sur la valorisation du bambou au Togo. Rev. Sci. Environ. Univ. Lomé Togo, 2, 527.

Kokou K., Nuto Y. \& Atsri H., 2009. Impact of charcoal production on woody plant species in West Africa: a case study in Togo. Sci. Res. Essay, 4(9), 881-893.

Koumantiga D., Wala K., Batawila K. \& Akpagana K., 2013. Les potentialités écotouristiques dans la préfecture de Doufelgou (Togo, Afrique de l'Ouest). Études caribéennes, 23.

Loubelo E., 2012. Impact des produits forestiers non ligneux (PFNL) sur l'économie des ménages et la sécurité alimentaire : cas de la République du Congo. Thèse de doctorat : Université Rennes 2 (France).

Luck G.W., Chan K. \& Fay J., 2009. Protecting ecosystem services and biodiversity in the world's watersheds. Conserv. Lett., 2, 179-188.

Mahamane A., Mahamane S. \& Lejoly J., 2007. Phénologie de quelques espèces ligneuses du parc national $\mathrm{du}$ 《 $\mathrm{W}$ » du Niger. Sécheresse, 18(4), 354-358.

MERF (Ministère de l'Environnement et des Ressources Forestières), 2016. Inventaire forestier national. Lomé : MERF.

Miller K., Allegreti M.H., Johnson N. \& Jonsson B., 1995. Measures for conservation of biodiversity and sustainable use of its components. In: Heywood V.H. \& Watson R.T., eds. Global biodiversity assessment. Cambridge, UK: Cambridge University Press, 915-1062.

Naito A.T. \& Cairns D.M., 2011. Relationships between Arctic shrub dynamics and topographically derived hydrologic characteristics. Environ. Res. Lett., 6(4), 045506 .
Neffati M., Sghaier M. \& Labbene Y., 2015. Rapport principal. Analyse de la vulnérabilité des écosystèmes et des moyens de subsistance (Livelihoods) des populations au changement climatique en zones arides et désertiques de la région MENA. Rabat : Centre national de documentation.

Ozier J., 1994. Endangered species and the private landowner: room for compatibility. J. For., 92(5), 22.

Rakotoarimanana V., Gondard H., Ranaivoarivelo N. \& Carriere S., 2008. Influence du pâturage sur la diversité floristique, la production et la qualité fourragères d'une savane des Hautes Terres malgaches (région de Fianarantsoa). Sécheresse, 19(1), 39-46.

Rives F., 2012. Gestion des forêts sèches à Madagascar et au Niger. Vulnérabilité et fonctions des systèmes socioécologiques pour comprendre les réformes forestières et leurs effets. Thèse de doctorat : AgroParisTech, Paris (France).

Salles J.M., 2010. Évaluer la biodiversité et les services écosystémiques: pourquoi, comment et avec quels résultats ? Nat.Sci.Soc., 18(4), 414-423.

Sop T.K. et al., 2011. Population structure of three woody species in four ethnic domains of the sub-Sahel of Burkina Faso. Land Degrad. Dev., 22, 519-529.

Traoré L., Ouédraogo I., Ouédraogo A. \& Thiombiano A., 2011. Perceptions, usages et vulnérabilité des ressources végétales ligneuses dans le Sud-Ouest du Burkina Faso. Int. J. Biol. Chem. Sci., 5(1), 258-278.

Van Auken O.W., 2009. Causes and consequences of woody plant encroachment into western North American grasslands. J. Environ. Manage., 90(10), 2931-2942.

Wala K., 2001. Typologie, structure et fonctionnement des agrosystèmes traditionnels dans la préfecture de Doufelgou (Nord-Togo). Mémoire : Université Nationale du Bénin, Cotonou (Bénin).

Yelkouni M., 2012. Gestion d'une ressource naturelle et action collective : le cas de la forêt de Tiogo au Burkina Faso. Thèse de doctorat: Université d'Auvergne, Clermont-Ferrand (France).

Zabouh K.,2014. Contribution à l'étude des plantes utilisées en tradimédecine vétérinaire dans la région des Savanes $d u$ Togo. Thèse de doctorat : Université de Lomé (Togo).

Zohoun G. et al., 2002. L'utilisation des produits forestiers non ligneux (PFNL) dans le cadre de la gestion forestière durable au Bénin. Le Flamboyant, 55, 13-18.

(55 réf.) 
Annexe 1. Liste exhaustive des espèces répertoriées et des usages connus dans la savane sèche - Comprehensive list of species and known uses in dry savannah.

\begin{tabular}{|c|c|c|c|c|}
\hline Numéro & Nom scientifique & Famille & $\begin{array}{l}\text { Nombre de } \\
\text { citations }\end{array}$ & Types d'usages connus/utilisations de l'espèce \\
\hline 1 & Acacia albida & Mimosaceae & 4 & $\begin{array}{l}\text { Bois-énergie, rituel, pharmaceutique, bois de } \\
\text { service }\end{array}$ \\
\hline 2 & Acacia gourmaensis & Mimosaceae & 5 & $\begin{array}{l}\text { Soins corporels, fourrage, pharmaceutique, } \\
\text { bois-énergie }\end{array}$ \\
\hline 3 & Acacia polyacantha & Mimosaceae & 4 & Fourrage \\
\hline 4 & Adansonia digitata & Bombacaceae & 151 & $\begin{array}{l}\text { Alimentaire, pharmaceutique, rituel, fourrage, } \\
\text { bois de service }\end{array}$ \\
\hline 5 & Afraegle paniculata & Rutaceae & 12 & Alimentaire, pharmaceutique, cosmétique \\
\hline 6 & Afzelia africana & Caesalpiniaceae & 68 & $\begin{array}{l}\text { Pharmaceutique, fourrage, rituel, artisanat, } \\
\text { bois-énergie, bois de service }\end{array}$ \\
\hline 7 & Albizia Lebbeck & Fabaceae & 12 & Fourrage, bois-énergie, bois de service \\
\hline 8 & Alchornea hirtella & Euphorbiaceae & 4 & Cosmétique \\
\hline 9 & Alchornea spp. & Euphorbiaceae & 1 & Pharmaceutique \\
\hline 10 & Anacardium occidentale & Anacardiaceae & 51 & $\begin{array}{l}\text { Alimentaire, bois-énergie, cosmétique, pharma- } \\
\text { ceutique }\end{array}$ \\
\hline 11 & Annona glauca & Annonaceae & 1 & Alimentaire \\
\hline 12 & Annona muricata & Annonaceae & 3 & Alimentaire \\
\hline 13 & Annona ondulata & Annonaceae & 2 & Bois-énergie \\
\hline 14 & Annona senegalensis & Annonaceae & 38 & Alimentaire, pharmaceutique, fourrage, rituel \\
\hline 15 & Annona squamosa & Annonaceae & 7 & Alimentaire \\
\hline 16 & Anogeissus leiocarpus & Combretaceae & 58 & $\begin{array}{l}\text { Bois-énergie, pharmaceutique, fourrage, rituel, } \\
\text { bois de service, rituel, artisanat, soins corporels }\end{array}$ \\
\hline 17 & Anthocleista djalonensis & Loganiaceae & 2 & Pharmaceutique \\
\hline 18 & Antiaris africana & Moraceae & 10 & $\begin{array}{l}\text { Bois-énergie, pharmaceutique, artisanat, rituel, } \\
\text { bois de service }\end{array}$ \\
\hline 19 & Artocarpus altilis & Moraceae & 1 & Alimentaire \\
\hline 20 & Artocarpus hétérophyllus & Moraceae & 1 & Alimentaire \\
\hline 21 & Azadirachta indica & Meliaceae & 173 & $\begin{array}{l}\text { Bois-énergie, soins corporels, pharmaceutique, } \\
\text { cosmétique, fourrage, artisanat, bois de service, } \\
\text { rituel }\end{array}$ \\
\hline 22 & Balanites aegyptiaca & Zygophyllaceae & 24 & Alimentaire, bois-énergie, fourrage \\
\hline 23 & Bambusa vulgaris & Poaceae & 4 & Bois de service \\
\hline 24 & Blighia sapida & Sapindaceae & 122 & $\begin{array}{l}\text { Alimentaire, bois-énergie, fourrage, soins } \\
\text { corporels, pharmaceutique, cosmétique, rituel }\end{array}$ \\
\hline 25 & Bombax costatum & Bombacaceae & 55 & $\begin{array}{l}\text { Alimentaire, phamaceutique, artisanat, fourrage, } \\
\text { rituel, cosmétique, bois de service }\end{array}$ \\
\hline 26 & Borassus aethiopum & Arecaceae & 130 & $\begin{array}{l}\text { Alimentaire, fourrage, soins corporels, bois- } \\
\text { énergie, cosmétique, artisanat, rituel, bois de } \\
\text { service }\end{array}$ \\
\hline 27 & Bridelia ferruginea & Euphorbiaceae & 4 & $\begin{array}{l}\text { Pharmaceutique, bois-énergie, artisanat, soins } \\
\text { corporels }\end{array}$ \\
\hline 28 & Burkea africana & Caesalpiniaceae & 13 & Soins corporels, bois de service, artisanat \\
\hline
\end{tabular}


Annexe 1 (suite 1). Liste exhaustive des espèces répertoriées et des usages connus dans la savane sèche - Comprehensive list of species and known uses in dry savannah.

\begin{tabular}{|c|c|c|c|c|}
\hline Numéro & Nom scientifique & Famille & $\begin{array}{l}\text { Nombre de } \\
\text { citations }\end{array}$ & Types d'usages connus/utilisations de l'espèce \\
\hline 29 & Carica papaya & Caricaceae & 45 & Alimentaire, pharmaceutique \\
\hline 30 & Carissa edulis & Apocynaceae & 3 & Alimentaire, pharmaceutique \\
\hline 31 & Cassia bomduc & Caesalpiniaceae & 1 & Fourrage \\
\hline 32 & Cassia sieberiana & Caesalpiniaceae & 4 & Pharmaceutique \\
\hline 33 & Ceiba pentandra & Bombacaceae & 97 & $\begin{array}{l}\text { Alimentaire, fourrage, pharmaceutique, } \\
\text { artisanat, rituel, bois de service, cosmétique, } \\
\text { bois-énergie }\end{array}$ \\
\hline 34 & Citrus aurantifolia & Rutaceae & 13 & Alimentaire, soins corporels \\
\hline 35 & Citrus limon & Rutaceae & 22 & Alimentaire, pharmaceutique \\
\hline 36 & Citrus maxima & Rutaceae & 2 & Alimentaire \\
\hline 37 & Citrus sinensis & Rutaceae & 57 & Alimentaire, pharmaceutique, bois de service \\
\hline 38 & Cocos nucifera & Arecaceae & 29 & $\begin{array}{l}\text { Alimentaire, pharmaceutique, cosmétique, } \\
\text { artisanat }\end{array}$ \\
\hline 39 & Cola niguritana & Sterculiaceae & 1 & Alimentaire \\
\hline 40 & Combretum aculeatum & Combretaceae & 5 & Pharmaceutique \\
\hline 41 & Combretum crotonoides & Combretaceae & 1 & Soins corporels \\
\hline 42 & Combretum glutinosum & Combretaceae & 7 & Pharmaceutique, bois-énergie \\
\hline 43 & Combretum spp. & Combretaceae & 5 & Bois-énergie \\
\hline 44 & Conocarpus erectus & Combretaceae & 1 & Pharmaceutique \\
\hline 45 & Crescentia cujete & Bignoniaceae & 3 & Artisanat \\
\hline 46 & Crossopteryx febrifuga & Rubiaceae & 9 & Bois-énergie, soins corporels \\
\hline 47 & Daniellia oliveri & Caesalpiniaceae & 44 & $\begin{array}{l}\text { Fourrage, bois-énergie, pharmaceutique, } \\
\text { artisanat, bois de service }\end{array}$ \\
\hline 48 & Delonix regia & Caesalpiniaceae & 3 & Pharmaceutique, rituel, bois de service \\
\hline 49 & Detarium microcarpum & Caesalpiniaceae & 39 & Alimentaire, pharmaceutique, bois-énergie \\
\hline 50 & Detarium senegalense & Caesalpiniaceae & 1 & Alimentaire \\
\hline 51 & Diospyros mespiliformis & Ebenaceae & 115 & $\begin{array}{l}\text { Alimentaire, bois-énergie, pharmaceutique, } \\
\text { soins corporels, artisanat, rituel, bois de service }\end{array}$ \\
\hline 52 & Dracaena arborea & Dracaenaceae & 3 & Rituel \\
\hline 53 & Elaeis guineensis & Arecaceae & 152 & $\begin{array}{l}\text { Alimentaire, fourrage, artisanat, bois de service, } \\
\text { bois-énergie, cosmétique, soins corporels, rituel, } \\
\text { pharmaceutique }\end{array}$ \\
\hline 54 & Entada abyssinica & Fabaceae & 1 & Soins corporels \\
\hline 55 & Entada africana & Fabaceae & 2 & Soins corporels, bois-énergie \\
\hline 56 & Eucaluptus camaldulensis & Myrtaceae & 32 & $\begin{array}{l}\text { Pharmaceutique, bois-énergie, soins corporels, } \\
\text { bois de service, artisanat, fourrage }\end{array}$ \\
\hline 57 & Eucalyptus tereticornis & Myrtaceae & 9 & Pharmaceutique, soins corporels, bois-énergie \\
\hline 58 & Euphorbia balsamifera & Euphorbiaceae & 1 & Pharmaceutique \\
\hline 59 & Faidherbia albida & Mimosaceae & 8 & Fourrage, pharmaceutique \\
\hline 60 & Feretia microcarpa & Rubiaceae & 1 & Pharmaceutique \\
\hline 61 & Ficus abutilifolia & Moraceae & 1 & Cosmétique \\
\hline
\end{tabular}


Annexe 1 (suite 2). Liste exhaustive des espèces répertoriées et des usages connus dans la savane sèche - Comprehensive list of species and known uses in dry savannah.

\begin{tabular}{|c|c|c|c|c|}
\hline Numéro & Nom scientifique & Famille & $\begin{array}{l}\text { Nombre de } \\
\text { citations }\end{array}$ & Types d'usages connus/utilisations de l'espèce \\
\hline 62 & Ficus capreifolia & Moraceae & 1 & Bois-énergie \\
\hline 63 & Ficus elastica & Moraceae & 1 & Fourrage \\
\hline 64 & Ficus exasperata & Moraceae & 4 & Pharmaceutique, rituel, bois-énergie \\
\hline 65 & Ficus glumosa & Moraceae & 11 & $\begin{array}{l}\text { Alimentaire, pharmaceutique, fourrage, soins } \\
\text { corporels }\end{array}$ \\
\hline 66 & Ficus polita & Moraceae & 31 & $\begin{array}{l}\text { Bois de service, bois-énergie, pharmaceutique, } \\
\text { fourrage }\end{array}$ \\
\hline 67 & Ficus spp. & Moraceae & 22 & Alimentaire, fourrage, rituel, pharmaceutique \\
\hline 68 & Ficus sur & Moraceae & 70 & Alimentaire, fourrage, pharmaceutique, bois-énergie \\
\hline 69 & Ficus sycomorus & Moraceae & 10 & Bois-énergie, cosmétique, fourrage, rituel \\
\hline 70 & Ficus thonningii & Moraceae & 34 & Fourrage, bois-énergie, bois de service, rituel \\
\hline 71 & Gardenia erubescens & Rubiaceae & 40 & Alimentaire, bois-énergie, pharmaceutique, rituel \\
\hline 72 & Gardenia sokotensis & Rubiaceae & 1 & Alimentaire \\
\hline 73 & Gardenia ternifolia & Rubiaceae & 4 & Cosmétique, rituel \\
\hline 74 & Gmelina arborea & Verbenaceae & 62 & $\begin{array}{l}\text { Fourrage, bois-énergie, pharmaceutique, bois de } \\
\text { service, artisanat }\end{array}$ \\
\hline 75 & Grewia flavescens & Tiliaceae & 1 & Rituel \\
\hline 76 & Grewia lasiodiscus & Tiliaceae & 8 & Alimentaire, fourrage \\
\hline 77 & Grewia mollis & Tiliaceae & 4 & Pharmaceutique \\
\hline 78 & Guiera senegalensis & Combretaceae & 1 & Pharmaceutique \\
\hline 79 & Gymnema sylvestre & Asclepiadaceae & 2 & Pharmaceutique \\
\hline 80 & Hannoa undulata & Simaroubaceae & 1 & Bois-énergie \\
\hline 81 & Holarrhena floribunda & Apocynaceae & 1 & Rituel \\
\hline 82 & Hymenocardia acida & Euphorbiaceae & 14 & Bois-énergie, rituel, soins corporels \\
\hline 83 & Hyphaene thebaica & Arecaceae & 48 & $\begin{array}{l}\text { Alimentaire, soins corporels, cosmétique, artisanat, } \\
\text { rituel, bois-énergie, pharmaceutique }\end{array}$ \\
\hline 84 & Irvingia gabonensis & Irvingiaceae & 1 & Alimentaire \\
\hline 85 & Isoberlinia doka & Caesalpiniaceae & 16 & Fourrage, bois-énergie, artisanat, bois de service \\
\hline 86 & Isoberlinia tomentosa & Lamiaceae & 5 & Alimentaire, bois-énergie, bois de service \\
\hline 87 & Jatropha curcas & Euphorbiaceae & 3 & Pharmaceutique, cosmétique \\
\hline 88 & Jatropha gossypiifolia & Euphorbiaceae & 1 & Pharmaceutique \\
\hline 89 & Khaya senegalensis & Meliaceae & 98 & $\begin{array}{l}\text { Pharmaceutique, bois-énergie, soins corporels, } \\
\text { fourrage, bois de service, artisanat, rituel }\end{array}$ \\
\hline 90 & Kigelia africana & Bignoniaceae & 1 & Rituel \\
\hline 91 & Lannea acida & Anacardiaceae & 22 & $\begin{array}{l}\text { Alimentaire, pharmaceutique, soins corporels, } \\
\text { fourrage, bois-énergie, cosmétique, bois de service }\end{array}$ \\
\hline 92 & Lannea barteri & Anacardiaceae & 19 & $\begin{array}{l}\text { Alimentaire, rituel, artisanat, pharmaceutique, bois- } \\
\text { énergie }\end{array}$ \\
\hline 93 & Lannea microcarpa & Anacardiaceae & 37 & $\begin{array}{l}\text { Alimentaire, pharmaceutique, fourrage, soins } \\
\text { corporels }\end{array}$ \\
\hline
\end{tabular}


Annexe 1 (suite 3). Liste exhaustive des espèces répertoriées et des usages connus dans la savane sèche - Comprehensive list of species and known uses in dry savannah.

\begin{tabular}{|c|c|c|c|c|}
\hline Numéro & Nom scientifique & Famille & $\begin{array}{l}\text { Nombre de } \\
\text { citations }\end{array}$ & $\begin{array}{l}\text { Types d'usages connus/utilisations de } \\
\text { l'espèce }\end{array}$ \\
\hline 94 & Lawsonia inermis & Lythraceae & 7 & Pharmaceutique, cosmétique, rituel \\
\hline 95 & Leucaena leucocephala & Mimosaceae & 16 & Fourrage, bois-énergie, bois de service \\
\hline 96 & Lippia multiflora & Verbenaceae & 1 & Alimentaire \\
\hline 97 & Lophira lanceolata & Ochnaceae & 3 & Pharmaceutique, bois de service \\
\hline 98 & Mangifera indica & Anacardiaceae & 236 & $\begin{array}{l}\text { Alimentaire, fourrage, pharmaceutique, } \\
\text { bois-énergie, bois de service, artisanat }\end{array}$ \\
\hline 99 & Margaritaria discoidea & Euphorbiaceae & 2 & Pharmaceutique, fourrage \\
\hline 100 & Milicia excelsa & Moraceae & 21 & $\begin{array}{l}\text { Rituel, bois de service, bois-énergie, } \\
\text { artisanat }\end{array}$ \\
\hline 101 & Millettia thonningii & Caesalpiniaceae & 2 & Pharmaceutique, artisanat \\
\hline 102 & Mitragyna inermis & Rubiaceae & 8 & $\begin{array}{l}\text { Pharmaceutique, artisanat, bois de service, } \\
\text { bois-énergie }\end{array}$ \\
\hline 103 & Moringa oleifera & Moringaceae & 76 & Alimentaire, pharmaceutique, fourrage \\
\hline 104 & Musa sp. & Musaceae & 2 & Pharmaceutique \\
\hline 105 & Newbouldia laevis & Bignoniaceae & 2 & Pharmaceutique \\
\hline 106 & Ocimum basilicum & Euphorbiaceae & 4 & Alimentaire, pharmaceutique \\
\hline 107 & Oncoba spinosa & Flacourtiaceae & 1 & Alimentaire \\
\hline 108 & Parinari curatellifolia & Chrysobalanaceae & 3 & Alimentaire, bois-énergie \\
\hline 109 & Parkia biglobosa & Mimosaceae & 208 & $\begin{array}{l}\text { Alimentaire, bois-énergie, pharmaceutique, } \\
\text { artisanat, rituel, soins corporels, cosmétique }\end{array}$ \\
\hline 110 & Pentadesma & Clusiaceae & 5 & Alimentaire, cosmétique \\
\hline 111 & Persea americana & Lauraceae & 4 & Alimentaire \\
\hline 112 & Piliostigma thonningii & Caesalpiniaceae & 16 & Pharmaceutique, bois-énergie, artisanat \\
\hline 113 & Prosopis africana & Mimosaceae & 130 & $\begin{array}{l}\text { Bois-énergie, soins corporels, } \\
\text { pharmaceutique, artisanat, bois de service, } \\
\text { fourrage, rituel }\end{array}$ \\
\hline 114 & Pseudocedrela kotschyi & Meliaceae & 40 & Soins corporels, pharmaceutique, artisanat \\
\hline 115 & Psidium guajava & Myrtaceae & 56 & $\begin{array}{l}\text { Alimentaire, pharmaceutique, soins } \\
\text { corporels }\end{array}$ \\
\hline 116 & Pteleopsis suberosa & Combretaceae & 38 & $\begin{array}{l}\text { Pharmaceutique, bois-énergie, soins } \\
\text { corporels }\end{array}$ \\
\hline 117 & Pterocarpus erinaceus & Fabaceae & 147 & $\begin{array}{l}\text { Fourrage, pharmaceutique, bois-énergie, } \\
\text { artisanat, bois de service, rituel, alimentaire, } \\
\text { cosmétique, soins corporels }\end{array}$ \\
\hline 118 & Raphia sudanica & Arecaceae & 3 & Bois de service, artisanat \\
\hline 119 & Saba comorensis & Apocynaceae & 6 & Soins corporels, alimentaire \\
\hline 120 & Saba senegalensis & Apocynaceae & 4 & Alimentaire, pharmaceutique \\
\hline 121 & Sarcocephalus latifolius & Rubiaceae & 45 & Pharmaceutique, alimentaire, fourrage \\
\hline 122 & Securidaca longepedunculata & Polygalaceae & 20 & Pharmaceutique, soins corporels, alimentaire \\
\hline 123 & Securinega virosa & Euphorbiaceae & 2 & Pharmaceutique \\
\hline 124 & Senna siamea & Caesalpiniaceae & 40 & Pharmaceutique, fourrage, bois-énergie \\
\hline
\end{tabular}


Annexe 1 (suite 4). Liste exhaustive des espèces répertoriées et des usages connus dans la savane sèche - Comprehensive list of species and known uses in dry savannah.

\begin{tabular}{|c|c|c|c|c|}
\hline Numéro & Nom scientifique & Famille & $\begin{array}{l}\text { Nombre de } \\
\text { citations }\end{array}$ & $\begin{array}{l}\text { Types d'usages connus/utilisations de } \\
\text { l'espèce }\end{array}$ \\
\hline 125 & Spondias mombin & Anacardiaceae & 3 & Alimentaire, pharmaceutique \\
\hline 126 & Sterculia setigera & Sterculiaceae & 12 & Pharmaceutique, alimentaire, artisanat, ritue \\
\hline 127 & Sterculia tragacantha & Sterculiaceae & 1 & Alimentaire \\
\hline 128 & Stereospermum kunthianum & Bignoniaceae & 6 & Fourrage, pharmaceutique \\
\hline 129 & Strychnos innocua & Loganiaceae & 14 & Alimentaire, pharmaceutique \\
\hline 130 & Strychnos spinosa & Loganiaceae & 10 & Alimentaire, pharmaceutique \\
\hline 131 & Swartzia madagascariensis & Caesalpiniaceae & 1 & Pharmaceutique \\
\hline 132 & Tamarindus indica & Caesalpiniaceae & 44 & $\begin{array}{l}\text { Alimentaire, pharmaceutique, soins } \\
\text { corporels, fourrage, cosmétique, bois de } \\
\text { service }\end{array}$ \\
\hline 133 & Tapinanthus & Loranthaceae & 1 & Artisanat \\
\hline 134 & Tectona grandis & Verbenaceae & 59 & $\begin{array}{l}\text { Pharmaceutique, cosmétique, artisanat, bois } \\
\text { de service, bois-énergie }\end{array}$ \\
\hline 135 & Terminalia glaucescens & Combretaceae & 12 & $\begin{array}{l}\text { Bois-énergie, soins corporels, cosmétique, } \\
\text { artisanat, bois de service }\end{array}$ \\
\hline 136 & Terminalia laxiflora & Combretaceae & 1 & Soins corporels \\
\hline 137 & Terminalia macroptera & Combretaceae & 9 & Cosmétique, fourrage \\
\hline 138 & Terminalia mollis & Combretaceae & 4 & Soins corporels \\
\hline 139 & Terminalia spp. & Combretaceae & 11 & Soins corporels, bois-énergie, artisanat \\
\hline 140 & Uvaria chamae & Annonaceae & 1 & Pharmaceutique \\
\hline 141 & Vernonia amygdalina & Asteraceae & 8 & Pharmaceutique, alimentaire \\
\hline 142 & Vernonia colorata & Asteraceae & 4 & Alimentaire, pharmaceutique \\
\hline 143 & Vitellaria paradoxa & Sapotaceae & 300 & $\begin{array}{l}\text { Alimentaire, cosmétique, pharmaceutique, } \\
\text { fourrage, bois-énergie, artisanat, rituel, bois } \\
\text { de service }\end{array}$ \\
\hline 144 & Vitex doniana & Verbenaceae & 97 & $\begin{array}{l}\text { Alimentaire, pharmaceutique, fourrage, } \\
\text { bois-énergie, artisanat }\end{array}$ \\
\hline 145 & Vitex simplicifolia & Verbenaceae & 5 & Alimentaire, pharmaceutique \\
\hline 146 & Xeroderris stuhlmannii & Fabaceae & 2 & Pharmaceutique \\
\hline 147 & Ximenia americana & Oleaceae & 2 & Alimentaire \\
\hline 148 & Zanthoxylum zanthoxyloides & Rutaceae & 14 & Alimentaire, pharmaceutique \\
\hline 149 & Ziziphus mисronatana & Rhamnaceae & 1 & Alimentaire \\
\hline Total & & & 4021 & \\
\hline
\end{tabular}


Annexe 2. Tableau exhaustif des valeurs d'importance des espèces (VIsp) répertoriées dans la savane sèche Comprehensive table of significance values for species (VIsp) listed in dry savanna.

\begin{tabular}{|c|c|c|}
\hline Numéro & Espèce & VIsp \\
\hline 1 & Acacia albida & 0,44 \\
\hline 2 & Acacia gourmaensis & 0,44 \\
\hline 3 & Acacia polyacantha & 0,11 \\
\hline 4 & Adansonia digitata & 0,55 \\
\hline 5 & Afraegle paniculata & 0,33 \\
\hline 6 & Afzelia africana & 0,66 \\
\hline 7 & Albizia lebbeck & 0,33 \\
\hline 8 & Alchornea hirtella & 0,11 \\
\hline 9 & Alchornea spp. & 0,11 \\
\hline 10 & Anacardium occidentale & 0,44 \\
\hline 11 & Annona glauca & 0,11 \\
\hline 12 & Annona muricata & 0,11 \\
\hline 13 & Annona ondulata & 0,11 \\
\hline 14 & Annona senegalensis & 0,44 \\
\hline 15 & Annona squamosa & 0,11 \\
\hline 16 & Anogeissus leiocarpus & 0,88 \\
\hline 17 & Anthocleista djalonensis & 0,11 \\
\hline 18 & Antiaris africana & 0,55 \\
\hline 19 & Artocarpus altilis & 0,11 \\
\hline 20 & Artocarpus hétérophyllus & 0,11 \\
\hline 21 & Azadirachta indica & 1,00 \\
\hline 22 & Balanites aegyptiaca & 0,33 \\
\hline 23 & Bambusa vulgaris & 0,11 \\
\hline 24 & Blighia sapida & 0,88 \\
\hline 25 & Bombax costatum & 0,77 \\
\hline 26 & Borassus aethiopum & 1,00 \\
\hline 27 & Bridelia ferruginea & 0,44 \\
\hline 28 & Burkea africana & 0,44 \\
\hline 29 & Carica papaya & 0,22 \\
\hline 30 & Carissa edulis & 0,22 \\
\hline 31 & Casia bomduc & 0,11 \\
\hline 32 & Cassia sieberiana & 0,11 \\
\hline 33 & Ceiba pentandra & 0,88 \\
\hline 34 & Citrus aurantifolia & 0,22 \\
\hline 35 & Citrus limon & 0,22 \\
\hline 36 & Citrus maxima & 0,11 \\
\hline 37 & Citrus sinensis & 0,33 \\
\hline 38 & Cocos nucifera & 0,44 \\
\hline
\end{tabular}

Annexe 2 (suite 1). Tableau exhaustif des valeurs d'importance des espèces (VIsp) répertoriées dans la savane sèche -Comprehensive table of significance values for species (VIsp) listed in dry savanna.

\begin{tabular}{|c|c|c|}
\hline Numéro & Espèce & VIsp \\
\hline 39 & Cola niguritana & 0,11 \\
\hline 40 & Combretum aculeatum & 0,11 \\
\hline 41 & Combretum crotonoides & 0,11 \\
\hline 42 & Combretum glutinosum & 0,22 \\
\hline 43 & Combretum spp. & 0,11 \\
\hline 44 & Conocarpus erectus & 0,11 \\
\hline 45 & Crescentia cujete & 0,11 \\
\hline 46 & Crossopteryx febrifuga & 0,22 \\
\hline 47 & Daniellia oliveri & 0,55 \\
\hline 48 & Delonix regia & 0,33 \\
\hline 49 & Detarium microcarpum & 0,33 \\
\hline 50 & Detarium senegalense & 0,11 \\
\hline 51 & Diospyros mespiliformis & 0,77 \\
\hline 52 & Dracaena arborea & 0,11 \\
\hline 53 & Elaeis guineensis & 1,00 \\
\hline 54 & Entada abyssinica & 0,11 \\
\hline 55 & Entada africana & 0,22 \\
\hline 56 & Eucaluptus camaldulensis & 0,66 \\
\hline 57 & Eucalyptus tereticornis & 0,33 \\
\hline 58 & Euphorbia balsamifera & 0,11 \\
\hline 59 & Faidherbia albida & 0,22 \\
\hline 60 & Feretia microcarpa & 0,11 \\
\hline 61 & Ficus abutilifolia & 0,11 \\
\hline 62 & Ficus capreifolia & 0,11 \\
\hline 63 & Ficus elastica & 0,11 \\
\hline 64 & Ficus exasperata & 0,33 \\
\hline 65 & Ficus glumosa & 0,44 \\
\hline 66 & Ficus polita & 0,44 \\
\hline 67 & Ficus spp. & 0,44 \\
\hline 68 & Ficus sur & 0,44 \\
\hline 69 & Ficus sycomorus & 0,44 \\
\hline 70 & Ficus thonningii & 0,44 \\
\hline 71 & Gardenia erubescens & 0,44 \\
\hline 72 & Gardenia sokotensis & 0,11 \\
\hline 73 & Gardenia ternifolia & 0,22 \\
\hline 74 & Gmelina arborea & 0,55 \\
\hline 75 & Grewia flavescens & 0,11 \\
\hline 76 & Grewia lasiodiscus & 0,22 \\
\hline
\end{tabular}


Annexe 2 (suite 2). Tableau exhaustif des valeurs d'importance des espèces (VIsp) répertoriées dans la savane sèche -Comprehensive table of significance values for species (VIsp) listed in dry savanna.

\begin{tabular}{|c|c|c|}
\hline Numéro & Espèce & VIsp \\
\hline 77 & Grewia mollis & 0,11 \\
\hline 78 & Guiera senegalensis & 0,11 \\
\hline 79 & Gymnema sylvestre & 0,11 \\
\hline 80 & Hannoa undulata & 0,11 \\
\hline 81 & Holarrhena floribunda & 0,11 \\
\hline 82 & Hymenocardia acida & 0,33 \\
\hline 83 & Hyphaene thebaica & 0,77 \\
\hline 84 & Irvingia gabonensis & 0,11 \\
\hline 85 & Isoberlinia doka & 0,44 \\
\hline 86 & Isoberlinia tomentosa & 0,33 \\
\hline 87 & Jatropha curcas & 0,22 \\
\hline 88 & Jatropha gossypiifolia & 0,11 \\
\hline 89 & Khaya senegalensis & 0,77 \\
\hline 90 & Kigelia africana & 0,11 \\
\hline 91 & Lannea acida & 0,77 \\
\hline 92 & Lannea barteri & 0,55 \\
\hline 93 & Lannea microcarpa & 0,44 \\
\hline 94 & Lawsonia inermis & 0,33 \\
\hline 95 & Leucaena leucocephala & 0,33 \\
\hline 96 & Lippia multiflora & 0,11 \\
\hline 97 & Lophira lanceolata & 0,22 \\
\hline 98 & Mangifera indica & 0,77 \\
\hline 99 & Margaritaria discoidea & 0,22 \\
\hline 100 & Milicia excelsa & 0,44 \\
\hline 101 & Millettia thonningii & 0,22 \\
\hline 102 & Mitragyna inermis & 0,44 \\
\hline 103 & Moringa oleifera & 0,33 \\
\hline 104 & Musa sp. & 0,11 \\
\hline 105 & Newbouldia laevis & 0,11 \\
\hline 106 & Ocimum basilicum & 0,22 \\
\hline 107 & Oncoba spinosa & 0,11 \\
\hline 108 & Parinari curatellifolia & 0,22 \\
\hline 109 & Parkia biglobosa & 1,00 \\
\hline 110 & Terminalia mollis & 0,11 \\
\hline 111 & Pentadesma & 0,22 \\
\hline 112 & Persea americana & 0,11 \\
\hline 113 & Piliostigma thonningii & 0,44 \\
\hline 114 & Prosopis africana & 0,77 \\
\hline
\end{tabular}

Annexe 2 (suite 3). Tableau exhaustif des valeurs d'importance des espèces (VIsp) répertoriées dans la savane sèche -Comprehensive table of significance values for species (VIsp) listed in dry savanna.

\begin{tabular}{|c|c|c|}
\hline Numéro & Espèce & VIsp \\
\hline 115 & Pseudocedrela kotschyi & 0,44 \\
\hline 116 & Psidium guajava & 0,33 \\
\hline 117 & Pteleopsis suberosa & 0,44 \\
\hline 118 & Pterocarpus erinaceus & 1,00 \\
\hline 119 & Raphia sudanica & 0,22 \\
\hline 120 & Saba comorensis & 0,22 \\
\hline 121 & Saba senegalensis & 0,22 \\
\hline 122 & Sarcocephalus latifolius & 0,33 \\
\hline 123 & Securidaca longepedunculata & 0,33 \\
\hline 124 & Securinega virosa & 0,11 \\
\hline 125 & Senna siamea & 0,44 \\
\hline 126 & Spondias mombin & 0,22 \\
\hline 127 & Sterculia setigera & 0,44 \\
\hline 128 & Sterculia tragacantha & 0,11 \\
\hline 129 & Stereospermum kunthianum & 0,22 \\
\hline 130 & Strychnos innocua & 0,22 \\
\hline 131 & Strychnos spinosa & 0,22 \\
\hline 132 & Swartzia madagascariensis & 0,11 \\
\hline 133 & Tamarindus indica & 0,66 \\
\hline 134 & Tapinanthus & 0,11 \\
\hline 135 & Tectona grandis & 0,55 \\
\hline 136 & Terminalia glaucescens & 0,66 \\
\hline 137 & Terminalia laxiflora & 0,11 \\
\hline 138 & Terminalia macroptera & 0,22 \\
\hline 139 & Terminalia spp. & 0,33 \\
\hline 140 & Uvaria chamae & 0,11 \\
\hline 141 & Vernonia amygdalina & 0,22 \\
\hline 142 & Vernonia colorata & 0,22 \\
\hline 143 & Vitellaria paradoxa & 1,00 \\
\hline 144 & Vitex doniana & 0,55 \\
\hline 145 & Vitex simplicifolia & 0,22 \\
\hline 146 & Xeroderris stuhlmannii & 0,11 \\
\hline 147 & Ximenia americana & 0,11 \\
\hline 148 & Zanthoxylum zanthoxyloides & 0,22 \\
\hline 149 & Ziziphus mucronatana & 0,11 \\
\hline
\end{tabular}

